\title{
An introduction to the composite element method applied to the vibration analysis of trusses
}

\author{
Marcos Arndt, Roberto Dalledone Machado and Mildred Ballin Hecke \\ Federal University of Paraná, Graduated Course in Numerical Methods for Engineering, Caixa Postal 19011 - \\ CEP 81531-990 - Curitiba, PR, Brazil \\ E-mail: arndt@cesec.ufpr.br; rdm@cesec.ufpr.br; mildred@cesec.ufpr.br
}

\begin{abstract}
This paper introduces a new type of Finite Element Method (FEM), called Composite Element Method (CEM). The CEM was developed by combining the versatility of the FEM and the high accuracy of closed form solutions from the classical analytical theory. Analytical solutions, which fulfil some special boundary conditions, are added to FEM shape functions forming a new group of shape functions. CEM results can be improved using two types of approach: $h$-version and $c$-version. The $h$-version, as in FEM, is the refinement of the element mesh. On the other hand, in the $c$-version there is an increase of degrees of freedom related to the classical theory ( $c$-dof). The application of CEM in vibration analysis is thus investigated and a rod element is developed. Some samples which present frequencies and vibration mode shapes obtained by CEM are compared to those obtained by FEM and by the classical theory. The numerical results show that CEM is more accurate than FEM for the same number of total degrees of freedom employed. It is observed in the examples that the $c$-version of CEM leads to a super convergent solution.
\end{abstract}

Keywords: Composite element method, vibration analysis, finite element method

\section{Introduction}

In structural engineering, the increase of lighter and stronger materials combined with technological improvements have played a major role in obtaining better performance from structures. In these cases, the dynamic effects on the structure response is significantly important and it becomes mandatory that a vibration analysis be performed within the project.

Due to its importance and the applicability in vibration analysis much research has been devoted to solution methods. Among them we should emphasize: the Classical Theory (CT) $[9,13]$; the Finite Element Method (FEM) [3,16]; Finite Strip Method (FSM) [21]; Boundary Element Method (BEM) [4,17]; Transference Matrix Method [2,11,12]; Dynamic Stiffness Matrix Method [1,10]; and Stochastic Analyses [8,14].

Recently, Zeng [18-20] presented a variation of the Finite Element Method (FEM), called Composite Ele- ment Method (or simply, Composite Method) with the objective of analyzing structural vibration.

In essence, this method puts together the versatility of FEM with the high precision of Classical Theory. In the CEM, analytical solutions from the classical theory, which comply with certain special boundary conditions, are added up to the shape functions of FEM creating, thus, new shape functions. For some specific applications, the same ideas behind CEM have been already presented in the literature as "extended finite element method" [23].

The CEM refinement can be divided into two distinct techniques:

- $h$-refinement

- c-refinement.

Numerical results have shown that the Composite Method is more accurate than the FEM with the same number of degrees of freedom. Moreover, the $c$ - 


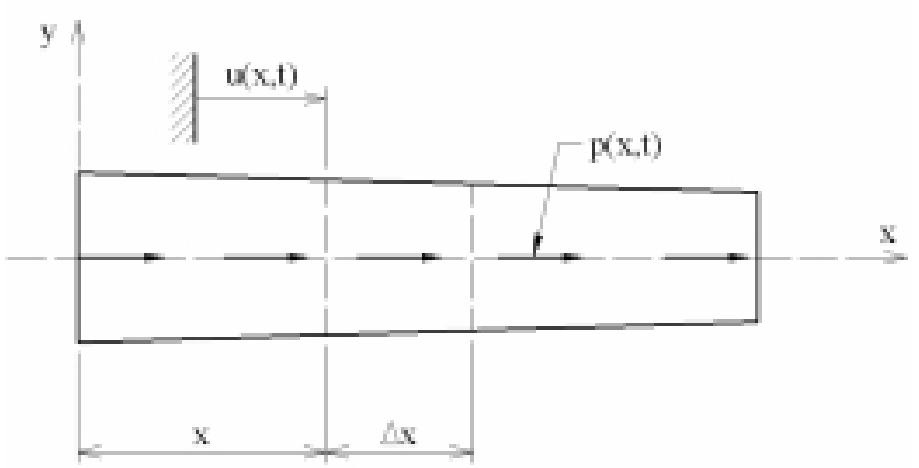

Fig. 1. Straight bar with axial deformation.

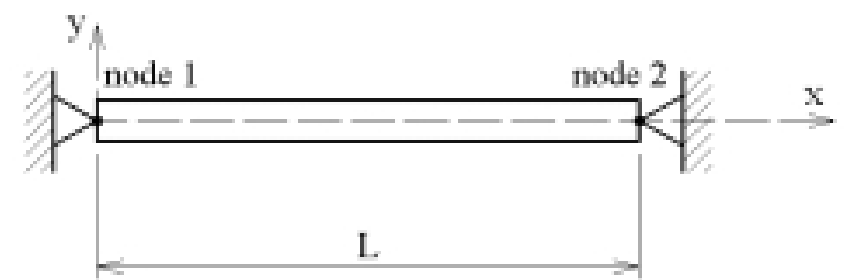

Fig. 2. Boundary conditions of the bar for interpolation functions of the CT.

refinement showed super convergence in the numerical solution results.

Despite the general applicability of the CEM to many engineering problems (as, for instance, the structural instability analysis), the present work aims, specifically, at the application of the Composite Method to vibration analysis.

\section{The composite element method}

The Composite Element Method, just as the FEM, consists of the domain partition into sub domains, named "elements", that are joined by nodes.

In solid mechanics problems, the element displacement field in the CEM is given by the nodal values from the FEM combined with the analytical solutions obtained in the element domain from the Classical Theory (CT). In order to achieve this, two coordinate systems are used:

- The Nodal Coordinate System - from the FEM;

- The Field Coordinate System - which is necessary to identify internal values from the CT.

The Nodal Coordinate System from the FEM gives the displacement field $u_{F E M}$ through the element nodal displacements:

$$
u_{F E M}(\xi)=\mathbf{N}^{T}(\xi) \mathbf{q}
$$

where, $\mathbf{N}$ is the shape function vector; $\mathbf{q}$ is the nodal displacement vector (or the nodal degrees of freedom); and $\xi$ is the element local coordinate.

In the Field Coordinate System, the displacement field $u_{C T}$ is given by coefficients that multiply functions of the Classical Theory, through the equation:

$$
u_{C T}(\xi)=\emptyset^{T}(\xi) \mathbf{c}
$$

where, $\varnothing$ is the analytical functions vector from the CT and $\mathbf{c}$ is the coefficient vector ( $c$ degrees of freedom or $c$ coordinates). The analytical functions from the CT are obtained through the solution of the differential equation, in the element domain, with compatible boundary conditions. The displacement field in the FEM (equation (1)) obeys nodal boundary conditions of the element. Therefore, it is necessary that the part from the CT (Eq. (2)) be analyzed by special boundary conditions, called compatibility boundary conditions, in order to maintain the values of those nodal displacements.

For that reason, the displacement field in the CEM is:

$$
u(\xi)=\mathbf{N}^{T}(\xi) \mathbf{q}+\emptyset^{T}(\xi) \mathbf{c}
$$



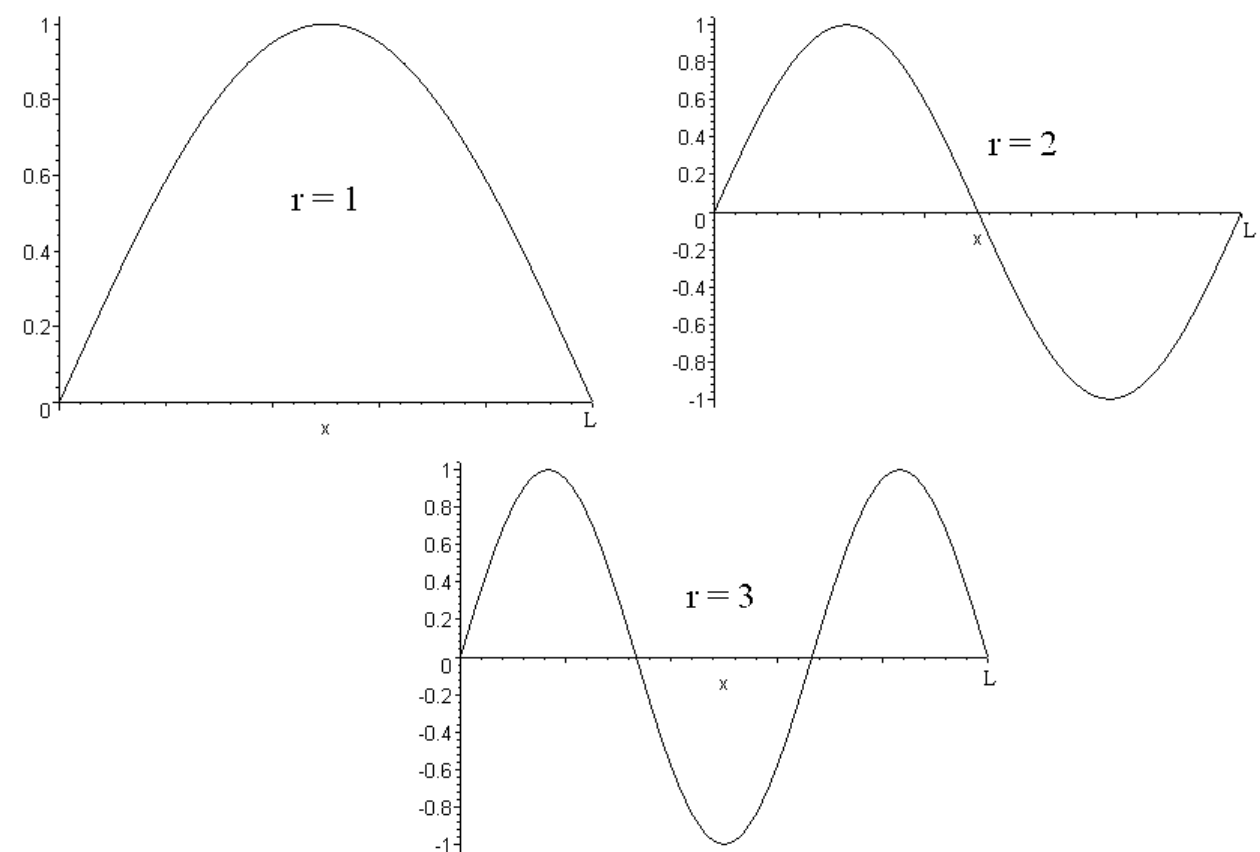

Fig. 3. Shape functions of the Classical Theory for the truss element to the first three vibration modes.

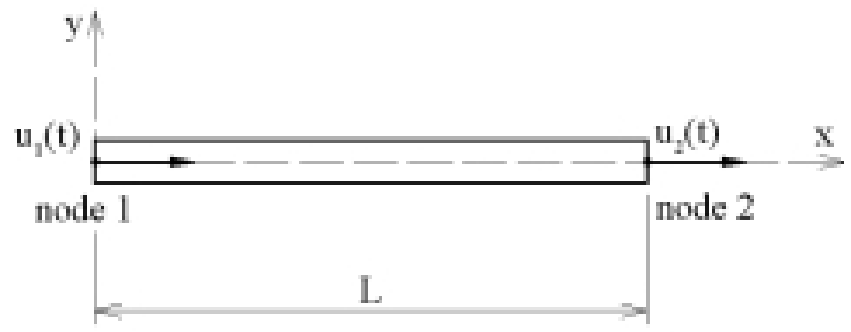

Fig. 4. Truss element.

Like the FEM, the CEM may be refined using an $h$-refinement technique. Moreover, as we have mentioned before, it is also possible to refine it through the $c$-refinement technique, which corresponds to a numerical increase of the analytical functions in the shape functions set, that is, an increase $c$-dof (from the Classical Theory). The later kind of refinement is hierarchic because, when increasing another term on the interpolation function, it is not necessary to recalculate the whole stiffness and mass matrix, except for the part related with the new degree of freedom (Carey and Oden [5]).

Aiming at analyzing the efficiency of the CEM, a uniform truss element for vibration analysis studies is developed in the next sections.

\section{Truss element}

The truss element corresponds to a straight bar with axial deformation (see Fig. 1) where the following hypothesis will be taken as relevant [7]:

a) the transversal section remains plane, perpendicular to the axis and with no rotation;

b) linear elastic material;

c) the properties of the material will be constant in transversal section, but it might vary in the longitudinal axis.

\subsection{Classical Theory}

The differential equation that governs the free vibration from the Classical Theory: 


$$
\frac{\partial}{\partial x}\left(E \cdot A \cdot \frac{\partial u}{\partial x}\right)-\rho \cdot A \cdot \frac{\partial^{2} u}{\partial t^{2}}=0
$$

where, $u(x, t)$ is the longitudinal displacement, $A$ is the transversal section area; $E$ is the Young's module and $\rho$ is the specific mass.

For obtaining natural frequencies and vibration modes, a harmonic movement is presumed in the bar, given by

$$
u(x, t)=U(x) \cdot \cos (\omega \cdot t-\alpha)
$$

where, $U(x)$ is the vibration mode, $\omega$ is the natural frequency and $\alpha$ is the phase angle. Thus, for the specific case of the uniform bar, where $E, A$ and $\rho$ are constants in the whole bar, the Eq. (4) is reduced to:

$$
\text { E.A. } \frac{d^{2} U}{d x^{2}}+\omega^{2} \cdot \rho \cdot A \cdot U=0
$$

which solution is:

$$
\begin{aligned}
U(x)= & a_{1} \cdot \cos \left(\sqrt{\frac{\omega^{2} \cdot \rho}{E}} \cdot x\right) \\
& +a_{2} \cdot \sin \left(\sqrt{\frac{\omega^{2} \cdot \rho}{E}} \cdot x\right)
\end{aligned}
$$

where $a_{1}$ and $a_{2}$ are constants.

For obtaining natural frequencies and vibration modes of the bar, and consequently the analytical function for the CEM, it is essential to define boundary conditions of the problem. In addition, boundary conditions that guarantee compatibility with the FEM are adopted, that is, conditions that do not change the nodal degrees of freedom. In order to achieve this compatibility it is necessary that the analytical solutions be null in the nodes of the element $(U(0)=0$ e $U(L)=0)$, as in Fig. 2. Therefore, the solution of this free vibration problem is:

$$
\begin{aligned}
& \omega_{r}=\frac{r . \pi}{L} \cdot \sqrt{\frac{E}{\rho}} \\
& U_{r}(x)=a_{r} \cdot \sin \left(\frac{r \cdot \pi}{L} \cdot x\right)
\end{aligned}
$$

where $L$ is the element length and $r=1,2,3, \ldots$

It can be seen that the bar has infinite solutions, hence the analytical solutions that might be used in the CEM are also infinite in number. Therefore, the displacement field of the truss element from the $\mathrm{CT}$ is:

$$
u_{C T}(x, t)=\varnothing^{T}(x) \cdot \mathbf{c}(t)
$$

where

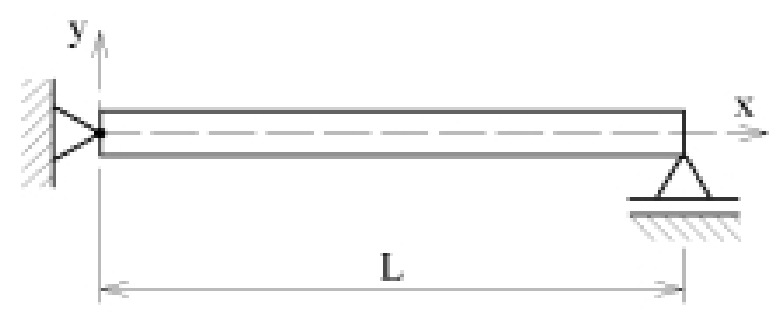

Fig. 5. Uniform straight bar.

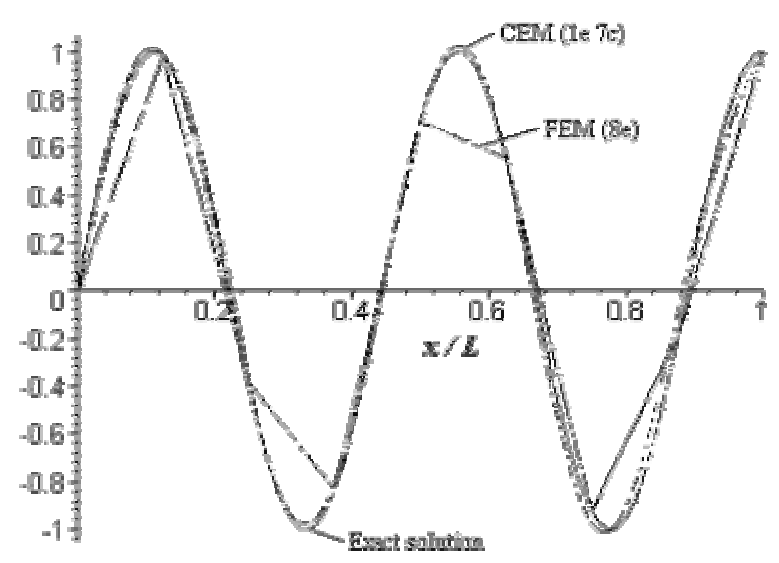

Fig. 6. Plot of fifth vibration mode equations.

$$
\begin{aligned}
& \emptyset^{T}(x)= {\left[\sin \left(\frac{\pi}{L} x\right) \sin \left(\frac{2 . \pi}{L} x\right)\right.} \\
&\left.\ldots \sin \left(\frac{n . \pi}{L} x\right)\right] \\
& \mathbf{c}^{T}(t)=\left[c_{1}(t) c_{2}(t) \ldots c_{n}(t)\right]
\end{aligned}
$$

and $n$ is the number of analytical functions.

The vector $\varnothing$ has shape functions of the CEM proceeding from the CT. The Fig. 3 shows the graphics of the first three shape functions of the CT.

\subsection{Finite element method}

Considering the uniform bar in Fig. (4) with one degree of freedom at each node, the displacement field is given by:

$$
u(x, t)=\psi_{1}(x) u_{1}(t)+\psi_{2}(x) u_{2}(t)
$$

where $\psi_{1}$ and $\psi_{2}$ are shape or interpolation functions, and $u_{1}$ and $u_{2}$ are the nodal displacements or nodal degrees of freedom.

When considering linear interpolation functions, the displacement field of the FEM for a uniform truss bar is $[3,6,22]$ :

$$
u_{F E M}(x, t)=\mathbf{N}^{T}(x) \cdot \mathbf{q}(t)
$$



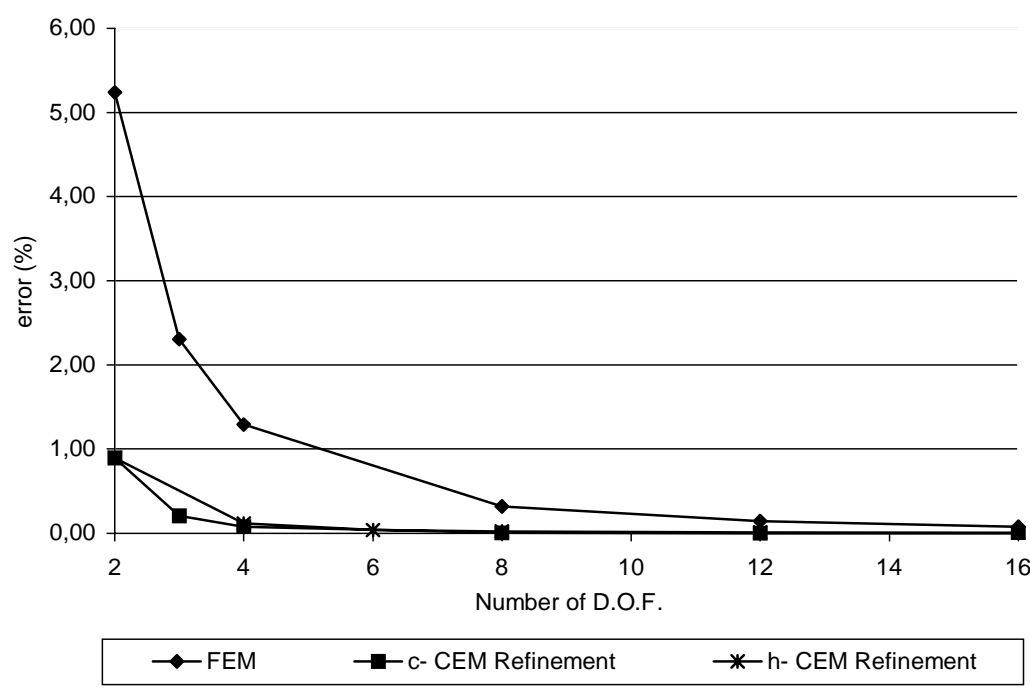

Fig. 7. Relative error (\%) for the first eingenvalue.

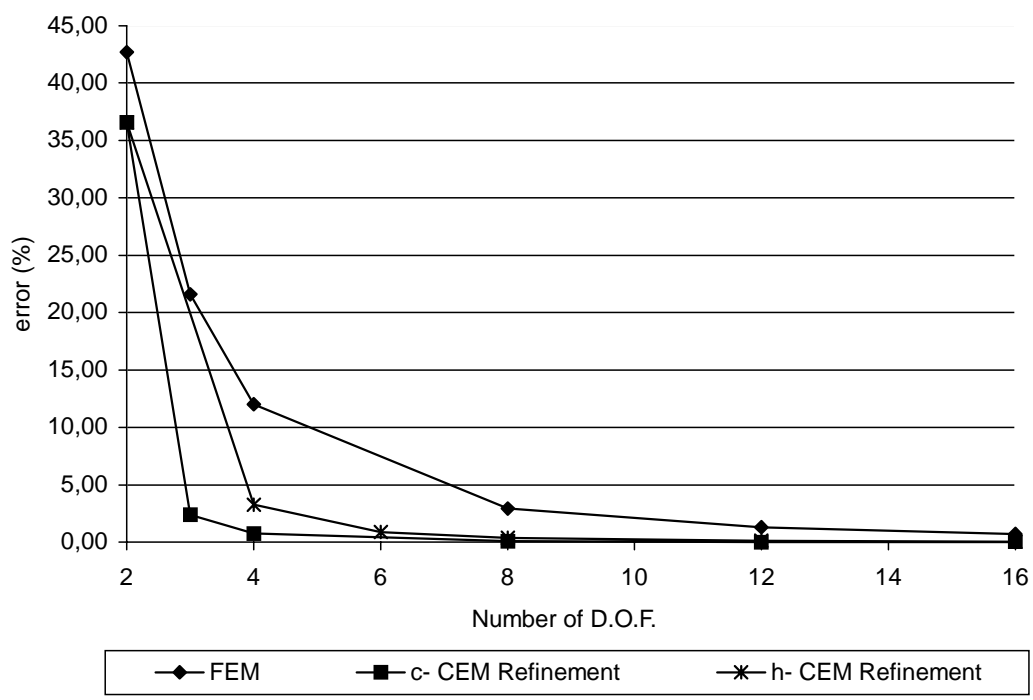

Fig. 8. Relative error (\%) for the second eingenvalue.

where:

$$
\begin{aligned}
& \mathbf{N}^{T}(x)=\left[\left(1-\frac{x}{L}\right) \frac{x}{L}\right] \\
& \mathbf{q}^{T}(t)=\left[u_{1}(t) u_{2}(t)\right]
\end{aligned}
$$

\subsection{Composite element method}

Considering the previous sections and recalling Eq. (3), the displacement field in the CEM for the uniform truss element is:

$$
u(x, t)=\mathbf{S}^{T}(x) \cdot \mathbf{v}(t)
$$

where

$$
\begin{gathered}
\begin{aligned}
\mathbf{S}^{T}(x)= & {\left[\left(1-\frac{x}{L}\right) \frac{x}{L} \sin \left(\frac{\pi}{L} x\right)\right.} \\
\left.\sin \left(\frac{2 . \pi}{L} x\right) \ldots \sin \left(\frac{n . \pi}{L} x\right)\right] & \\
\text { and } \mathbf{v}^{T}(t)= & {\left[u_{1}(t) u_{2}(t) c_{1}(t)\right.} \\
& \left.c_{2}(t) \ldots c_{n}(t)\right]
\end{aligned}
\end{gathered}
$$

where $n$ is the number of analytical functions or shape functions of the CT.

The axial deformation $(\varepsilon)$, might be written in the following way: 


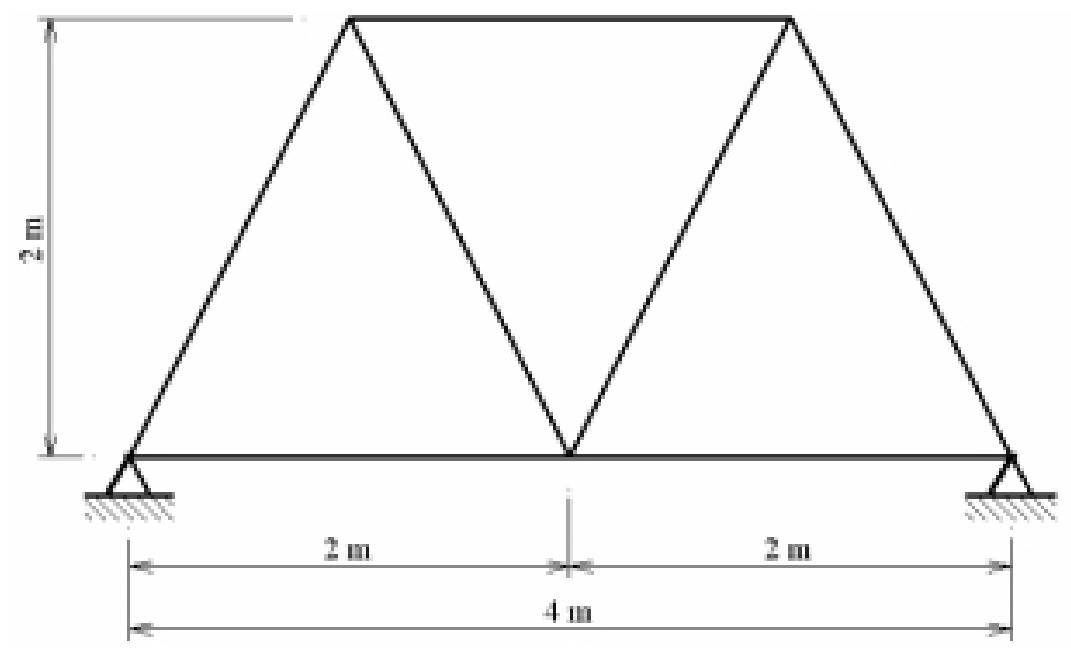

Fig. 9. Plane truss.

Table 1

Results from the FEM and CEM with 8 degrees of freedom

\begin{tabular}{|c|c|c|c|c|c|c|c|c|c|}
\hline \multirow[t]{2}{*}{$i$} & \multirow{2}{*}{$\begin{array}{l}\text { Exact } \\
\lambda_{i}^{2}\end{array}$} & \multicolumn{2}{|c|}{ FEM (8e) } & \multicolumn{2}{|c|}{ CEM (1e 7c) } & \multicolumn{2}{|c|}{ CEM (2e 3c) } & \multicolumn{2}{|c|}{ CEM (4e 1c) } \\
\hline & & $\lambda_{i}^{2}$ & $\begin{array}{c}\text { Error } \\
(\%)\end{array}$ & $\lambda_{i}^{2}$ & $\begin{array}{c}\text { Error } \\
(\%) \\
\end{array}$ & $\lambda_{i}^{2}$ & $\begin{array}{c}\text { Error } \\
(\%)\end{array}$ & $\lambda_{i}^{2}$ & $\begin{array}{c}\text { Error } \\
(\%) \\
\end{array}$ \\
\hline 1 & 2,467 & 2,475 & 0,322 & 2,468 & 0,008 & 2,468 & 0,014 & 2,468 & 0,021 \\
\hline 2 & 22,207 & 22,856 & 2,924 & 22,223 & 0,073 & 22,256 & 0,223 & 22,288 & 0,366 \\
\hline 3 & 61,685 & 66,780 & 8,260 & 61,817 & 0,213 & 62,095 & 0,664 & 62,816 & 1,834 \\
\hline 4 & 120,903 & 140,808 & 16,464 & 121,449 & 0,452 & 121,841 & 0,776 & 126,922 & 4,979 \\
\hline 5 & 199,859 & 254,259 & 27,219 & 201,541 & 0,841 & 202,831 & 1,487 & 233,628 & 16,896 \\
\hline 6 & 298,556 & 413,547 & 38,516 & 303,059 & 1,508 & 313,002 & 4,839 & 384,988 & 28,950 \\
\hline 7 & 416,991 & 601,854 & 44,333 & 429,004 & 2,881 & 554,565 & 32,992 & 598,517 & 43,532 \\
\hline 8 & 555,165 & 746,282 & 34,425 & 796,169 & 43,411 & 818,672 & 47,465 & 799,670 & 44,042 \\
\hline
\end{tabular}

Notes: FEM (ne) - Finite Element Method with $n$ elements. CEM (ne mc) - Composite Element Method with $n$ elements and $m c$-dof for each element.

$$
\varepsilon=\mathbf{B}^{T}(x) \cdot \mathbf{v}(t)
$$

where:

$$
\begin{aligned}
& \mathbf{B}^{T}(x) \\
= & {\left[-\frac{1}{L} \frac{1}{L} \frac{\pi}{L} \cdot \cos \left(\frac{\pi}{L} x\right) \frac{2 \pi}{L}\right.} \\
& \left.\cos \left(\frac{2 \pi}{L} x\right) \ldots \frac{n \pi}{L} \cdot \cos \left(\frac{n \pi}{L} x\right)\right]
\end{aligned}
$$

Applying the Virtual Work Principle, one obtains:

$$
\mathbf{m} . \ddot{\mathbf{v}}(t)+\mathbf{k} \cdot \mathbf{v}(t)=\mathbf{0}
$$

where: $\ddot{\mathbf{v}}(t)=\frac{d^{2}}{d t^{2}}[\mathbf{v}(t)]$

$$
\begin{aligned}
& \mathbf{k}=E \cdot A \cdot \int_{0}^{L} \mathbf{B} \cdot \mathbf{B}^{T} \cdot d x \\
& \mathbf{m}=\rho \cdot A \cdot \int_{0}^{L} \mathbf{S} \cdot \mathbf{S}^{T} \cdot d x
\end{aligned}
$$

where $\mathbf{k}$ is the stiffness matrix and $\mathbf{m}$ the consistent mass matrix of the element.

Assuming harmonic movement, Eq. (22), which should be valid for any time $t$, is transformed into the generalized eingenvalue problem:

$$
\left(\mathbf{k}-\omega^{2} \cdot \mathbf{m}\right) \cdot \mathbf{U}=\mathbf{0}
$$

where $\omega$ is the natural frequency (eingenvalue) and $\mathbf{U}$ is the natural vibration mode (eingenvector) composed by the nodal displacements and by the $c$ coordinates, in the following form:

$$
\mathbf{U}=\left[\begin{array}{llllll}
u_{1} & u_{2} & c_{1} & c_{2} & \ldots & c_{n}
\end{array}\right]^{T}
$$

Therefore, after integration, the bar elementary stiffness matrix can be obtained through:

$$
\mathbf{k}=E . A .\left[\begin{array}{l:l}
\frac{\mathbf{k}_{a a}}{\mathbf{k}_{b a}}: \frac{\mathbf{k}_{a b}}{\mathbf{k}_{b b}}
\end{array}\right]
$$

with: 
Table 2

Results obtained through the FEM and the CEM for the plane truss

\begin{tabular}{cccccc}
\hline I & $\begin{array}{c}\text { FEM }(7 \mathrm{e}) \\
\varpi_{i}(\mathrm{rad} / \mathrm{s})\end{array}$ & $\begin{array}{c}\text { CEM }(7 \mathrm{e} 1 \mathrm{c}) \\
\varpi_{i}(\mathrm{rad} / \mathrm{s})\end{array}$ & $\begin{array}{c}\text { Zeng }(7 \mathrm{e} 1 \mathrm{c}) \\
\varpi_{i}(\mathrm{rad} / \mathrm{s})\end{array}$ & $\begin{array}{c}\text { CEM }(7 \mathrm{e} 2 \mathrm{c}) \\
\varpi_{i}(\mathrm{rad} / \mathrm{s})\end{array}$ & $\begin{array}{c}\text { Zeng }(7 \mathrm{e} 2 \mathrm{c}) \\
\varpi_{i}(\mathrm{rad} / \mathrm{s})\end{array}$ \\
\hline 1 & 1683,521413 & 1648,516148 & 1648,52 & 1648,258910 & 1648,26 \\
2 & 1776,278483 & 1741,661466 & 1741,66 & 1741,319206 & 1741,32 \\
3 & 3341,375203 & 3119,123132 & 3119,12 & 3113,835167 & 3113,83 \\
4 & 5174,353866 & 4600,595156 & 4600,60 & 4567,688849 & 4567,69 \\
5 & 5678,184561 & 4870,575795 & 4870,58 & 4829,702095 & 4829,70 \\
6 & 8315,400602 & 7380,832845 & 7380,83 & 7379,960217 & 7379,96 \\
7 & & 8047,936309 & 8047,93 & 7532,305498 & 7532,30 \\
8 & & 8272,611818 & 8272,61 & 8047,936313 & 8047,93 \\
9 & & 11167,56472 & 11167,57 & 9997,484917 & 9997,48 \\
10 & & 12051,89683 & 12051,90 & 10567,42895 & 10567,43 \\
11 & & 14359,30988 & 14359,31 & 12282,63058 & 12282,63 \\
12 & & 15525,68547 & 15525,68 & 13296,29795 & 13296,29 \\
13 & & 16792,68173 & 16792,68 & 13654,89423 & 13654,89 \\
\hline
\end{tabular}

Notes: CEM (ne mc) - CEM with $n$ elements and $m c$-dof for each element; FEM (ne): FEM with $n$ elements; Zeng (ne mc) - results obtained by Zeng [18].

$$
\begin{aligned}
\mathbf{k}_{a a}= & {\left[\begin{array}{cc}
\frac{1}{L} & -\frac{1}{L} \\
-\frac{1}{L} & \frac{1}{L}
\end{array}\right] } \\
\mathbf{k}_{b b}= & {\left[\begin{array}{cccc}
\frac{\pi^{2}}{2 L} & 0 & \ldots & 0 \\
0 & \frac{4 \pi^{2}}{2 L} & \ldots & 0 \\
\vdots & \vdots & \ddots & \vdots \\
0 & 0 & \ldots & \frac{n^{2} \pi^{2}}{2 L}
\end{array}\right] }
\end{aligned}
$$

The sub matrix $\mathbf{k}_{a a}$ of matrix $\mathbf{k}$ corresponds to the elementary stiffness matrix of the FEM for the truss element. The sub matrices $\mathbf{k}_{a b}$ and $\mathbf{k}_{b a}$ are nulls and the sub matrix $\mathbf{k}_{b b}$ is the diagonal matrix of the CEM, for this element. It must be emphasized that the contribution from the analytical terms from the CT in the $\mathbf{k}$ matrix corresponds, uniquely, to the coefficients of sub matrix $\mathbf{k}_{b b}$. Moreover, there is no correspondence between the degrees of freedom from the FEM and the CEM.

Similarly, after integration, the consistent elementary mass matrix of the bar can be obtained through:

$$
\mathbf{m}=\rho . A .\left[\begin{array}{lll}
\mathbf{m}_{a a} & \mid \mathbf{m}_{a b} \\
\text { symmetric } & \mathbf{m}_{b b}
\end{array}\right]
$$

with:

$$
\begin{aligned}
\mathbf{m}_{a a} & =\left[\begin{array}{cc}
\frac{L}{3} & \frac{L}{6} \\
\frac{L}{6} & \frac{L}{3}
\end{array}\right] \\
\mathbf{m}_{b b}= & {\left[\begin{array}{cccc}
\frac{L}{2} & 0 & \ldots & 0 \\
0 & \frac{L}{2} & \ldots & 0 \\
\vdots & \vdots & \ddots & \vdots \\
0 & 0 & \ldots & \frac{L}{2}
\end{array}\right] }
\end{aligned}
$$

$$
\mathbf{m}_{a b}=\left[\begin{array}{cccc}
\frac{L}{\pi} & \frac{L}{2 \pi} & \cdots & \frac{L}{n \pi} \\
\frac{L}{\pi} & -\frac{L}{2 \pi} & \cdots & \frac{(-1)^{n+1} \cdot L}{n \pi}
\end{array}\right]
$$

The sub matrix $\mathbf{m}_{a a}$ of matrix $\mathbf{m}$ corresponds to the consistent mass matrix of the FEM for truss element. It can be noted that the $\mathbf{m}_{a b}$ e $\mathbf{m}_{b a}$ are not null matrices, that is, they must be evaluated and operated.

\section{Numerical verification}

For the numerical verification of the CEM, the free longitudinal vibration of the uniform straight bar (Fig. 5), with length $L$, elasticity modulus $E$, density $\rho$ and cross section area $A$, is analyzed.

Considering the boundary conditions of the problem, the exact solution is $[7,15]$ :

$$
\begin{aligned}
& \omega_{i}=(2 . i-1) \cdot \frac{\pi}{2 . L} \cdot \sqrt{\frac{E}{\rho}} \\
& U_{i}(x)=a \cdot \sin \left[(2 . i-1) \cdot \frac{\pi}{2 . L} \cdot x\right], \\
& \text { for } i=1,2, \ldots
\end{aligned}
$$

where $a$ is a constant and $U$ is the vibration mode of the bar.

The truss element shown in the last section was used for solving the problem through the CEM. For the solution through the FEM, however, the number of analytical functions ( $c$-dof) had to be made equal to zero.

In order to compare the exact solution with the solutions obtained from the CEM and from the FEM, the following form was used as a parameter: 

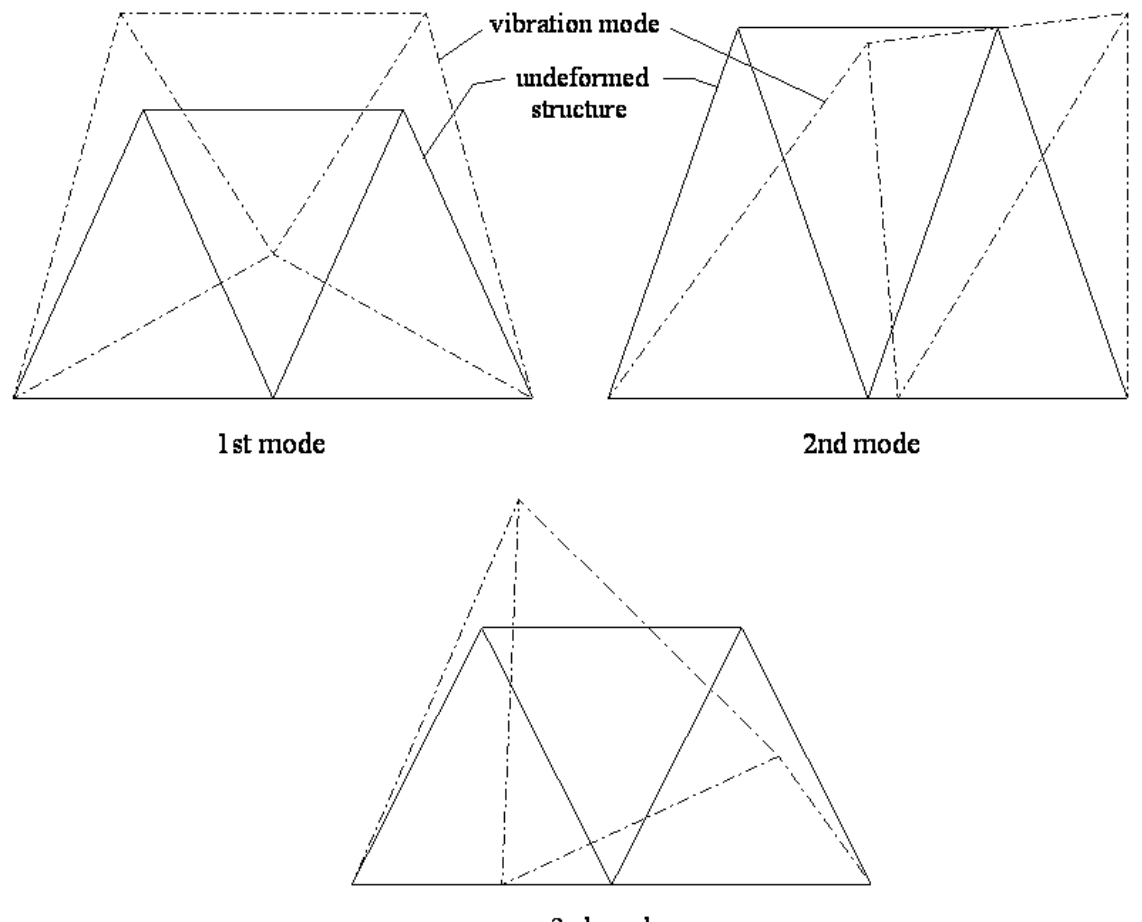

Fig. 10. The first three natural vibration modes for the plane truss CEM (7e 2c).

$$
\lambda_{i}^{2}=\frac{\rho . L^{2}}{E} \cdot \omega_{i}^{2}
$$

where $\omega_{i}$ is the natural frequency vibration of the bar.

The Table 1 shows the results obtained from the FEM and from the CEM for a model with a total number of degrees of freedom equal to 8 , that is, with the same computational effort.

The table shows that the first eingenvalues obtained through the CEM (and corresponding to the total numbers of $c$-dof) are more accurate than the corresponding eingenvalues from the FEM. On the other hand, however, the last eingenvalues from the CEM (corresponding to the number of nodal degrees of freedom) might be even worse than those obtained through the FEM with the same total number of degrees of freedom.

In Fig. 6 the equation of the fifth mode of vibration of bar obtained by the CEM, the FEM and also by the CT with 8 degrees of freedom are plotted.

The relative errors obtained for some eingenvalues $\lambda_{i}^{2}$ (Eq. (36)) through the FEM ( $h$-refinement) and through the $h$ and $c$ refinements of the CEM are shown in Figs 7 and 8.

It shows that the $c$-refinement from the CEM is more efficient than the $h$ one and shows super convergence in the results. The eingenvalues from the CEM corre- sponding to the number $c$-dof are more accurate than the eigenvalues from the FEM.

In order to obtain the 7th eingenvalue with relative error lower than 5\%, the CEM could be used with 1 element and $7 c$-dof (which corresponds to a total of 8-dof). Nevertheless, using the FEM with 16 elements, that is, a total of 16 degrees of freedom brings about a relative error of $14.15 \%$.

\section{Application}

In this application the free vibration of a seven bar truss (Fig. 9) is considered. Every single bar has the following characteristics: transversal section area $A=$ $0.001 \mathrm{~m}^{2}$; elasticity modulus $E=2.1 \times 10^{11} \mathrm{~N} / \mathrm{m}^{2}$; and specific mass $\rho=8000 \mathrm{Ns}^{2} / \mathrm{m}^{4}$. Table 2 shows the natural frequencies obtained through the FEM and the CEM. The results are compared to those obtained by Zeng [18].

There is an excellent agreement between Zeng's results [18] and those obtained in this study. Figure 10, for example, shows the first three natural vibration modes of the truss obtained through the CEM with 7 elements and $2 c$-dof for element. 


\section{Conclusion}

The combination between the FEM and the CT generates the CEM, a more accurate numerical method with the same versatility and efficiency of the FEM.

The stiffness and mass matrices of the truss element are symmetric and formed with particular characteristics. The stiffness matrix is formed by a sub matrix that corresponds to the stiffness matrix of the FEM, by a diagonal matrix of the $\mathrm{CT}$ and null components. The consistent mass matrix preserves the corresponding sub matrix of the consistent mass matrix of the FEM and a sub matrix from the CT. However it still has a sub matrix formed by terms that refers to the association between the FEM and the CEM.

The CEM developed in this study has two types of refinement (with boundary conditions of the analytical solutions chosen appropriately): $h$-refinement and $c$-refinement. The $h$-refinement, like the FEM, corresponds to the increase in the number of degrees of freedom through the increase in the number of elements that are part of the mesh. On the other hand, the $c$ refinement corresponds to the increase in the number $c$-dof obtained through the enrichment of the interpolation function. This enrichment does not alter the terms of the stiffness and mass matrices, which were previously calculated, it just aggregates new terms. It can be inferred, thus, that the $c$-refinement is hierarchic. The interpolation functions of the CEM for the element of this study are complete and consistent, that is, they have a high convergence in the results.

Comparing those refinements, it can be remarked that both of them have great accuracy, but the $c$-refinement is not only more efficient, but also has higher convergence of results. The CEM, hence, is more efficient when using the easiest mesh that represents the geometry with the appropriated number of $c$-dof.

In addition, the CEM is more accurate than the FEM with the same total number of dof for the majority of obtained frequencies. In the examples developed in this study, the frequencies have shown great accuracy (very close to the exact solution and much more accurate than the FEM with the same total number of degrees of freedom, that is, with the same computational cost). The values obtained through the CEM tend to be better than those obtained through the FEM, even over the first and the $n$-th frequency interval, where $n$ is the number of $c$-dof of CEM. In some cases though, some of those values, especially in the last frequencies, were less accurate than the FEM. Nevertheless, due to the su- per convergence property, if the $c$-refinement technique was used, the results would be a lot more accurate.

The tests have shown that, when aiming at great precision for a certain range of results, the model calculated by the CEM has a lower computational cost, that is, a lower total number of dof than a similar model calculated through the FEM.

We can get to the conclusion, therefore, that the CEM is a very accurate and powerful method for vibration analysis.

\section{References}

[1] J.R. Banerjee, Dynamic stiffness formulation for structural elements: a general approach, Computers \& Structures 63(1) (1997), 101-103.

[2] C.N. Bapat, Vibration of rods with uniformly tapered sections, Journal of Sound and Vibration 185(1) (1995), 185-189.

[3] K. Bathe, Finite element procedures, Prentice Hall, New Jersey, USA, 1996.

[4] C.A. Brebbia and D. Nardini, Dynamic analysis in solid mechanics by an alternative boundary element procedure, Soil Dynamics and Earthquake Engineering 2(4) (1983), 228-233.

[5] G.F. Carey and J.T. Oden, Finite elements, Vol. 3: Computational aspects, Prentice-Hall, New Jersey, USA, 1984.

[6] A.K. Chopra, Dynamics of structures: theory and applications to earthquake engineering, Prentice Hall, New Jersey, USA, 1995, pp. 729.

[7] R.R. Craig, Structural dynamics: an introduction to computer methods, John Wiley, New York, USA, 1981, pp. 527.

[8] M. Grigoriu, Stochastic mechanics, International Journal of Solids and Structures 37 (2000), 197-214.

[9] B.M. Kumar and R.I. Sujith, Exact solutions for the longitudinal vibration of non-uniform rods, Journal of Sound and Vibration 207(5) (1997), 721-729.

[10] A.Y.T. Leung and W.E. Zhou, Dynamic stiffness analysis of axially loaded non-uniform Timoshenko columns, Computers \& Structures 56(4) (1995), 577-588.

[11] Q.S. Li, Free longitudinal vibration analysis of multi-step nonuniform bars based on piecewise analytical solutions, Engineering Structures 22 (2000), 1205-1215.

[12] Q.S. Li, Exact solutions for free longitudinal vibration of stepped non-uniform rods, Applied Acoustics 60 (2000), 1328.

[13] Q.S. Li, H. Cao and G. Li, Static and dynamic analysis of straight bars with variable cross-section, Computers Structures 59(6) (1996), 1185-1191.

[14] Q.S. Li, J.Q. Fang and D.K. Liu, Evaluation of structural dynamic responses by stochastic finite element method, Structural Engineering and Mechanics 8(5) (1999), 477-490.

[15] L. Meirovicth, Elements of vibration analysis, Mc Graw-Hill, Tokio, Japan, pp. 495.

[16] R.K. Singh and H.A. Smith, Comparison of computational effectiveness of the finite element formulations in free vibration analyses, Computers \& Structures 51(4) (1975), 381-391.

[17] M. Tanaka, T. Matsumoto and A. Shiozaki, Application of boundary-domain element method to the free vibration problem of plate structures, Computers \& Structures 66(6) (1998), $725-735$. 
[18] P. Zeng, Composite element method for vibration analysis of structures, part I: principle and $\mathrm{C}^{0}$ element (bar), Journal of Sound and Vibration 218(4) (1998), 619-658.

[19] P. Zeng, Composite element method for vibration analysis of structures, part II: $\mathrm{C}^{1}$ element (beam), Journal of Sound and Vibration 218(4) (1998), 659-696.

[20] P. Zeng, Introduction to composite element method for structural analysis in engineering, Key Engineering Materials
145-149 (1998), 185-190.

[21] W.X. Zhong, Y.K. Cheung and Y. Li, The precise finite strip method, Computers and Structures 69 (1998), 773-783.

[22] O.C. Zienkiewicz, The finite element method, (3 ed.), McGraw-Hill, London, England, 1977.

[23] T. Belytschko and T. Brack, Elastic Crack Growth in Finite Elements with Minimal Remeshing, Int. J. Numer. Meth. Engng. 45 (1999), 601-620. 

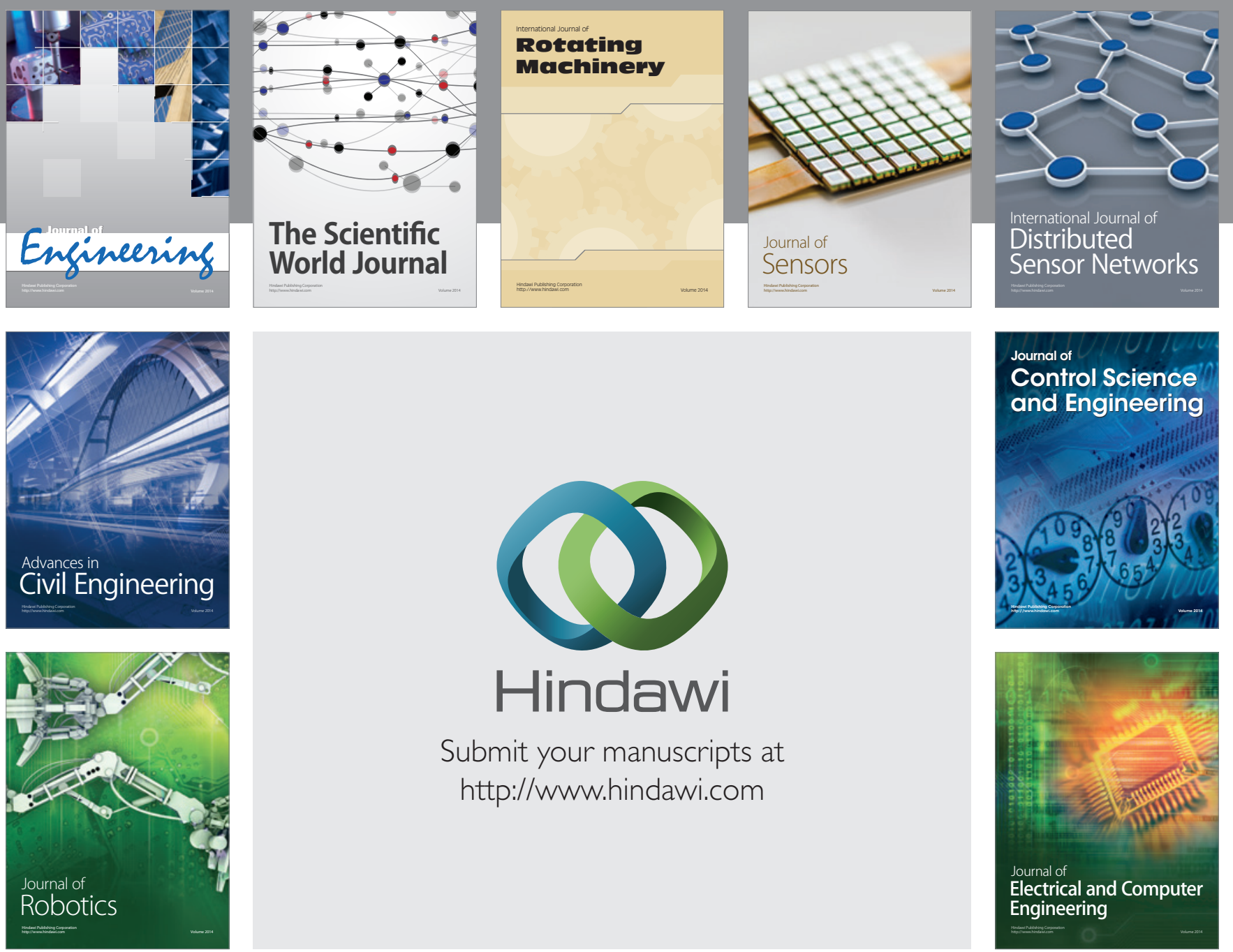

Submit your manuscripts at

http://www.hindawi.com
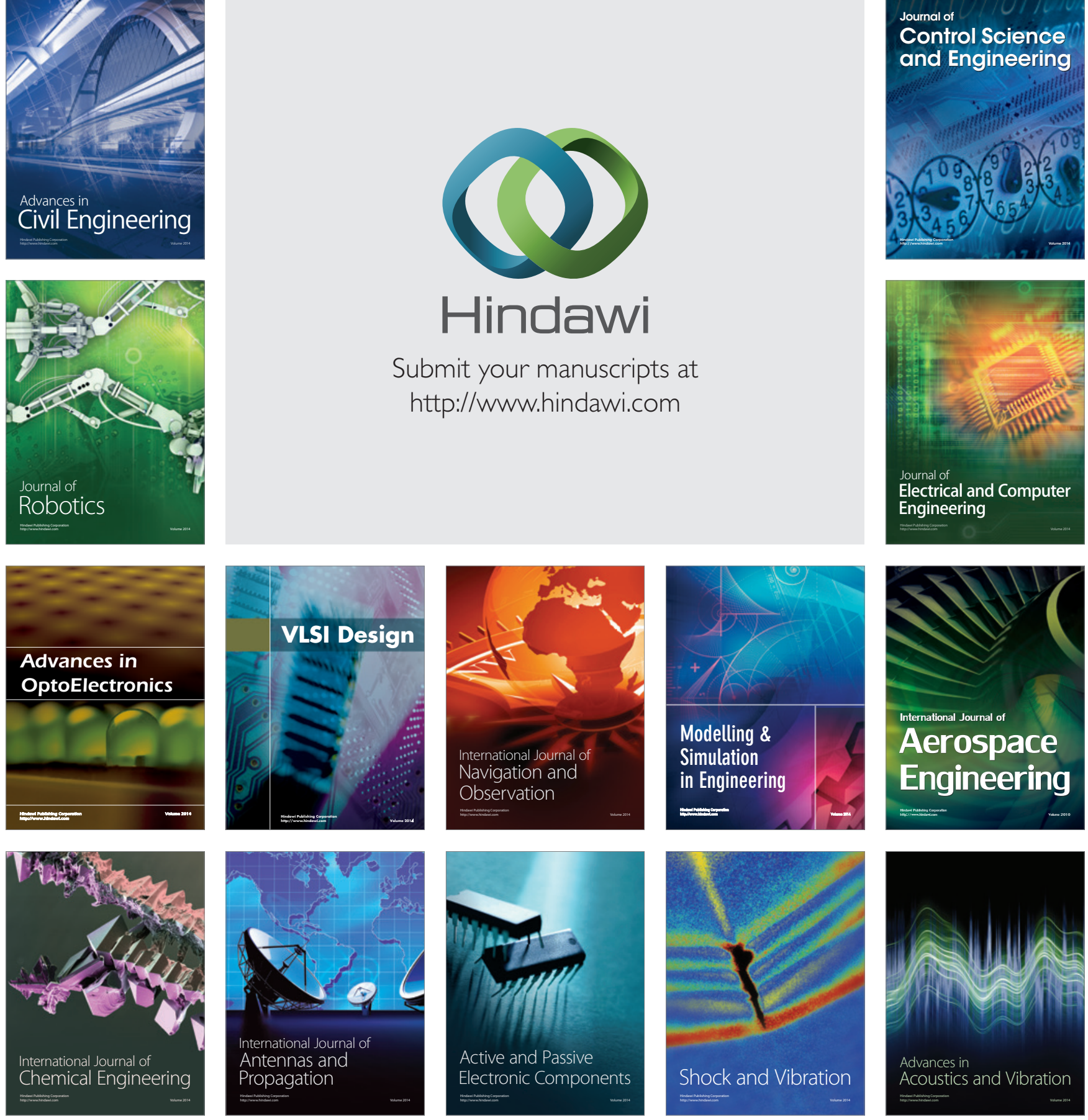